\title{
Preparation of silazane grafted yttria-stabilized zirconia nanocrystals via water/CTAB/hexanol reverse microemulsion
}

\author{
Clifford Y. Tai *, Bor-Yuan Hsiao, Hsien-Yi Chiu \\ Department of Chemical Engineering, National Taiwan University, No.1, Sec. 4, Roosevelt Road, Taipei, 106 Taiwan
}

Received 24 May 2006; accepted 31 May 2006

Available online 28 June 2006

\begin{abstract}
The nanosized precursors of yttria-stabilized zirconia (YSZ) were prepared via a water/CTAB/hexanol reverse microemulsion. The surface hydroxyl groups of the YSZ precursor were substituted by siloxyl groups using HMDS (hexamethydisilazane) as starting material. The untreated and treated YSZ powder were characterized by ultraviolet-visible-near-infrared spectrometer, FT-IR spectrometer, X-ray diffractometer, BET surface-area analyzer, and electron probe microanalysis (EPMA). After HMDS treatment, the amount of Si atom grafted on the nanoparticle surface was about $1 \mathrm{~mol} \%$. The crystallite size of the treated powder was smaller and the surface area was larger than the untreated powder. (C) 2006 Elsevier B.V. All rights reserved.
\end{abstract}

Keywords: Ceramics; Nanomaterials; $\mathrm{ZrO}_{2}$; Calcination; Grafting

\section{Introduction}

Zirconia powder is useful in many advanced structural, hightemperature and electrical ceramic applications. Zirconia has three crystalline phases including cubic, tetragonal, and monoclinic. Among these structures, the monoclinic phase is a thermodynamically stable structure at low temperature. The tetragonal phase of zirconia, however, is of great interest because of its application [1-3]. The doping with $\mathrm{Mg}^{2+}, \mathrm{Ca}^{2+}$, and $\mathrm{Y}^{3+}$ could stabilize tetragonal zirconia at a low temperature. For example, the yttria-stabilized zirconia (YSZ) has been widely used in oxygen sensors. Numerous techniques have been applied to produce pure or stabilizing additive doped zirconia nanocrystalline, including sol-gel [4], hydrothermal [5], and precipitation processing [6]. The precipitation technique is a convenient, safe, and inexpensive process, but it produces particles with a wider size distribution and irregular particle shape that reduce the quality of ceramics. It seems to us that these problems can be overcome by the reverse microemulsion precipitation technique. Reverse microemulsion solution is a transparent, isotropic and stable system with nanosized droplets dispersed in a continuous oil phase.

\footnotetext{
* Corresponding author.

E-mail address: cytai@ntu.edu.tw (C.Y. Tai).
}

The water droplets can be treated as nanosized reactors for the formation of nanoparticles [7]. Recently, the nanoparticles of zirconia precursor were successfully synthesized via a reverse microemulsion route [8-11].

To achieve fully crystalline nanocrystals of zirconia, a further calcination process is necessary. However, the crystal sizes of YSZ nanoparticles are sensitive to a firing process that would increase the crystal size at an elevating temperature or with a prolonged heat treatment. Hence, special attention should be paid to the control of crystal size and crystal structure during calcination. In the literature HMDS (hexamethyldisilazane) has been utilized to inhibit crystal growth. It can replace hydroxyl groups to form noncondensing methyl siloxyl surface groups, and they decompose in air at $350{ }^{\circ} \mathrm{C}$ to produce $\mathrm{SiO}_{2}$ particles that serve as the "pinning" particles for the inhibition of crystal growth [12]. The aim of this work is to compare the properties of two kinds of YSZ powder prepared via a reverse microemulsion process without and with a post treatment using HMDS.

\section{Experimental}

In this experiment, all the raw materials were used as received. Zirconyl nitrate, yttrium nitrate, surfactant CTAB (Cetytrimethyl ammonium bromide), and HMDS (hexamethyldisilazane) were 


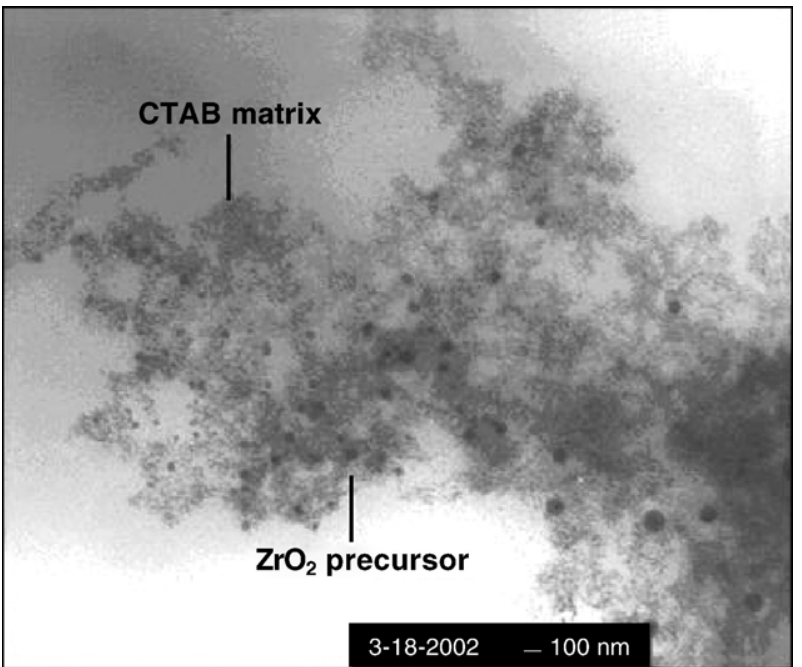

Fig. 1. TEM micrograph of YSZ precursors prepared by microemulsion precipitation for $S=0.3, W=0.2$. $S$ and $W$ are the weight ratio of surfactant-tooil and water-to-oil respectively.

purchased from Aldrich and aqueous ammonia solution (28 wt.\%) and 1-Hexanol (98.9\%) were from Nacalai Tesque and TEDIA, respectively.

For comparison two kinds of YSZ powder were prepared, i.e., with and without HMDS treatment. The procedures for the two types of powder were the same until the hydrous YSZ was produced. The first step was to prepare a reverse microemulsion by mixing a certain amount of hexanol, surfactant, and aqueous solution of yttrium and zirconyl nitrate in a $500 \mathrm{ml}$ jacket reactor. Then this reverse microemulsion with the mole ratio of $0.06 \mathrm{Y}^{3+}$ / $0.94 \mathrm{Zr}^{4+}$ was added dropwise into the reverse microemulsion of ammonia water with vigorous magnetic-stirring to obtain a slurry of YSZ precursor. The YSZ precursor was separated from the oil phase by centrifugation and further washed with water, ethanol (99.5\%), respectively, and dried at $80^{\circ} \mathrm{C}$ over $12 \mathrm{~h}$. At this stage, the product was referred to as hydrous YSZ powder. Then the hydrous YSZ powder was subjected to the HMDS treatment and was further calcined at various temperatures for $1 \mathrm{~h}$ to obtain the

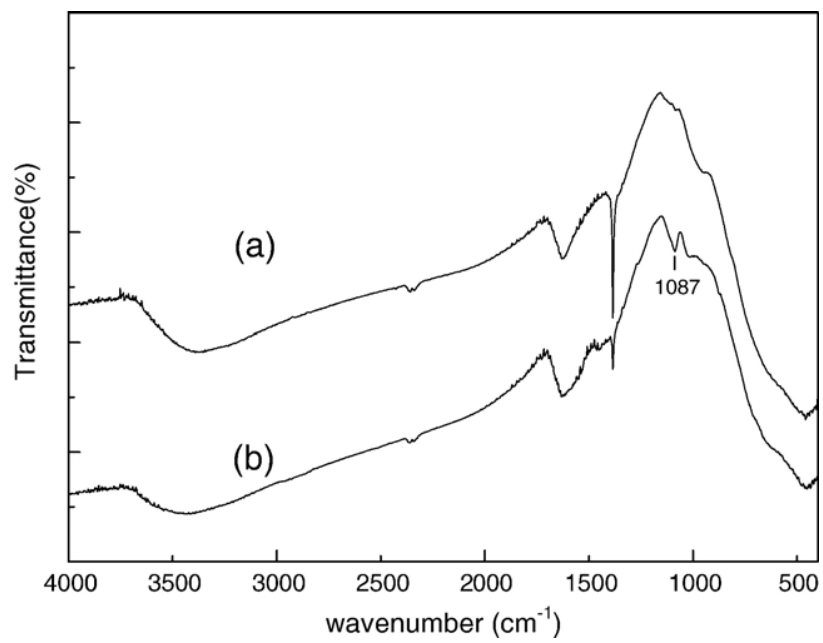

Fig. 2. FT-IR spectra of (a) untreated and (b) HMDS treated YSZ powder calcined at $650{ }^{\circ} \mathrm{C}$.

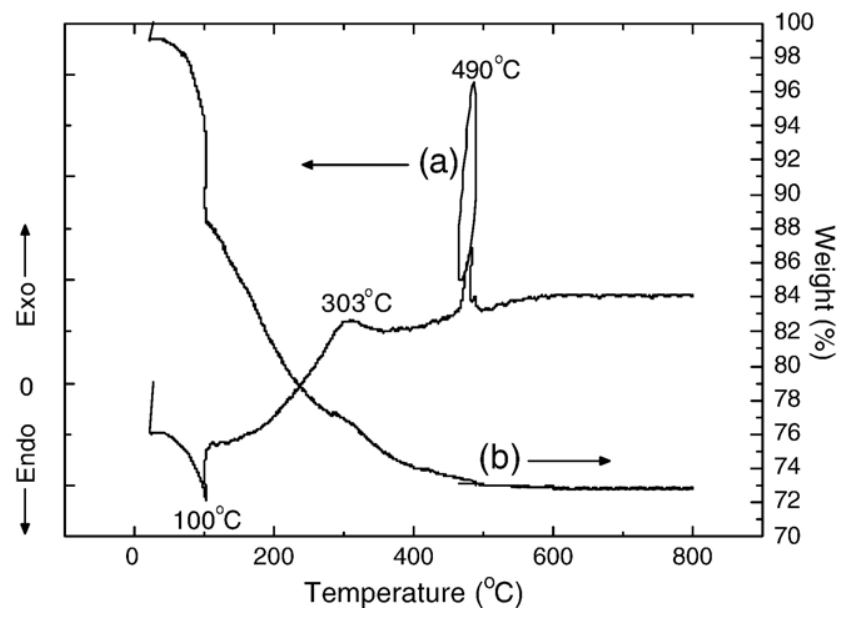

Fig. 3. TG/DTA analysis of YSZ precursors, (a) DTA curve and (b) TGA curve, heating rate at $10{ }^{\circ} \mathrm{C} / \mathrm{min}$.

fully crystalline YSZ nanocrystals. The HMDS treatment of hydrous YSZ powder was conducted at $150^{\circ} \mathrm{C}$ for $1 \mathrm{~h}$ in a vacuum vessel, following the procedures used by $\mathrm{Wu}$ et al. [12].

The particle size and morphology of the resultant YSZ nanoparticles were examined by a transmission electron microscope (TEM, Hitachi-7100). The crystal structure of the product powder was determined by an X-ray diffractometer (Mac Science, MXP-3 TXT-7266). The mean zirconia crystallite size was calculated from the Debye-Scherrer equation based on the full width at half maximum intensity of the $t_{(101)}$ peak for $t-\mathrm{ZrO}_{2}$. The specific surface area (BET) of the samples was determined from physical adsorption of $\mathrm{N}_{2}$ at $T=78 \mathrm{~K}$, measured in a Micrometritics ASAP 2010 analyzer. Ultraviolet-visible-near-infrared (UV-VISNIR) spectra were obtained at room temperature, measured by a Hitachi model U3410 spectrometer. Chemical bonding and functional groups were investigated with a Fourier transform infrared spectroscopy (FT-IR, Bio-Rad model FTS-40) and the FT-IR spectrum was collected after 16 scans with a normal resolution of $4 \mathrm{~cm}^{-1}$. The weight loss and reaction evolution of the powder were determined with a thermal analyzer (TGD-7000RH, Ulvac/

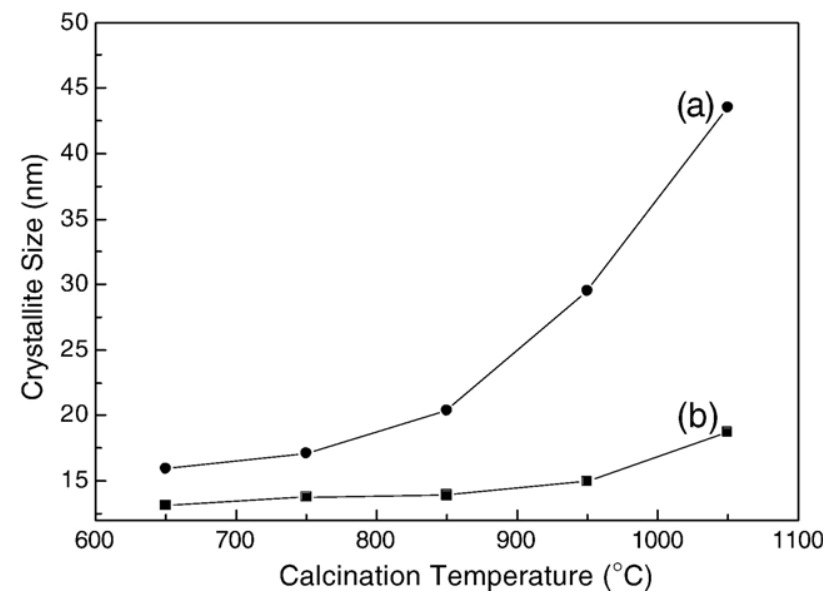

Fig. 4. Mean crystallite size of YSZ nanoparticles prepared in this experiment for (a) untreated sample, and (b) HMDS treated sample. 
Riko). The samples were heated from $25{ }^{\circ} \mathrm{C}$ to $1000{ }^{\circ} \mathrm{C}$ with a heating rate of $10^{\circ} \mathrm{C} / \mathrm{min}$ in a static air atmosphere.

\section{Results and discussion}

\subsection{Particle characterization}

A stable reverse microemulsion of water/CTAB/hexanol system can exist in a certain area on the three-component phase diagram as reported by Lufimpadio et al. [13]. In this experiment $0.25 \mathrm{M} \mathrm{ZrO}\left(\mathrm{NO}_{3}\right)_{2}$ and $6 \mathrm{~N} \mathrm{NH}_{4} \mathrm{OH}$ aqueous solution instead of pure water were used. The stable region of our system was slightly narrower than that of pure water. Then the two stable solutions of reverse microemulsion were mixed to form particles. The TEM micrograph of the YSZ precursors produced is shown in Fig. 1. The precursor nanoparticles, which had a spherical morphology and a particle size range of $10-15 \mathrm{~nm}$, were dispersed in CTAB matrix.

To detect the possible substitution of hydroxyl groups by the methyl siloxyl groups on the YSZ precursor before and after HMDS treatment, the samples were first examined by an ultraviolet-visible-near-infrared spectra analyzer. The $-\mathrm{OH}$ groups had strong absorption in the bands at 1180,1390, and $1720 \mathrm{~nm}$ for the untreated samples [12]. After HMDS treatment, the $-\mathrm{OH}$ absorption intensities at those bands were reduced. The samples were further subjected to FT-IR spectra analysis. Fig. 2 shows the FT-IR spectra of untreated and HMDS treated YSZ powder calcined at $650{ }^{\circ} \mathrm{C}$. The band at $1087 \mathrm{~cm}^{-1}$ on curve (b) indicated the formation of $\mathrm{Zr}-\mathrm{O}-\mathrm{Si}$ bond in the HMDS treated sample $[14,15]$. We concluded that some of the $-\mathrm{OH}$ bonds on the precursor were replaced by the $\mathrm{O}-\mathrm{Si}$ bonds after HMDS treatment.

\subsection{TG/DTA analysis}

To obtain fully crystalline nanoparticles of YSZ, the microemulsion-derived YSZ precursors need further calcination at higher temperatures. Calcination of the precursor particles burned out residual surfactant and nitrate group, and then the precursor particles transformed into tetragonal phase under suitable calcination conditions. Fig. 3 shows the curves of TG/DTA analysis for the precursor after heat treatment at a heating rate of $10{ }^{\circ} \mathrm{C} / \mathrm{min}$, in which curve (a) for the microemulsion-derived zirconia precursor presents three major peaks: one for endotherm and the other two for exotherm. The endothermic peak at about $100{ }^{\circ} \mathrm{C}$ corresponded to the elimination of residual water and alcohols. The broad exothermic peak at about $303{ }^{\circ} \mathrm{C}$ on the DTA curve was probably due to the oxidation of organic and nitrate group residues. The latter peak at $490{ }^{\circ} \mathrm{C}$, called the "glow exotherm", was attributed to the crystallization of zirconia from amorphous and hydrous forms $[9,16]$. According to the results of the TGA analysis shown in curve (b) on Fig. 3, the weight dropped to $73 \mathrm{wt} . \%$, and levelled off at $600{ }^{\circ} \mathrm{C}$. Therefore, calcination temperatures used in this experiment were higher than $600{ }^{\circ} \mathrm{C}$ to burn out impurities completely.

\subsection{Effect of HMDS treatment on particle size}

The mean crystallite sizes of untreated and HMDS treated YSZ powder determined by the Debye-Scherrer equation are plotted in Fig. 4 by applying the XRD diffraction patterns. The crystallite size of the untreated powder, shown in curve (a), increased rapidly from $16 \mathrm{~nm}$ to $42 \mathrm{~nm}$ when the calcination temperature changed from $650{ }^{\circ} \mathrm{C}$ to $1100^{\circ} \mathrm{C}$.
In contrast to the untreated powder, the crystallite size of the HMDS treated powder was essentially maintained below $15 \mathrm{~nm}$ in the temperature range from $650{ }^{\circ} \mathrm{C}$ to $950{ }^{\circ} \mathrm{C}$, as shown in curve (b). The measured BET (Brunauer-Emmett-Teller) specific surface areas were also consistent with the XRD observation. The specific surface areas of the untreated samples decreased rapidly from $84 \mathrm{~m}^{2} / \mathrm{g}$ to $29 \mathrm{~m}^{2} / \mathrm{g}$ in the temperature range of calcination between $550{ }^{\circ} \mathrm{C}$ and $850{ }^{\circ} \mathrm{C}$. The BET results of HMDS treated YSZ powder dropped from $169 \mathrm{~m}^{2} / \mathrm{g}$ to $102 \mathrm{~m}^{2} /$ $\mathrm{g}$ in the same temperature range. From the XRD and BET analysis, it is clear that the advantage of HMDS treatment is to prevent the YSZ nanocrystals from growing and thus maintain high surface area.

\section{Conclusions}

The yttria-stabilized zirconia precursors were prepared by the reverse microemulsion precipitation technique using a water/CTAB/hexanol system. The precursors were spherical in shape with a particle size range about $10-15 \mathrm{~nm}$ and were dispersed well in the surfactant matrix. The precursors were treated with hexamethyldisilazane (HMDS) so that the siloxyl groups grafted on the surface of YSZ precursors formed $\mathrm{Zr}-\mathrm{O}$ $\mathrm{Si}$ bonds after calcinations. The EPMA results showed that the amount of doped Si was about $1 \mathrm{~mol} \%$. The crystallite sizes of the HMDS-treated YSZ powder were kept smaller and rather uniform by adsorbing the siloxyl groups during calcination and thus the surface area remained high, when calcination was conducted below $1000{ }^{\circ} \mathrm{C}$.

\section{Acknowledgement}

This study was supported by the National Science Council of Taiwan.

\section{References}

[1] R. Stevens, Zirconia and Zirconia Ceramics, Report from Magnesium Elektron Ltd., Twickenham, 1986, p. 9.

[2] M. Mamak, N. Coombs, G. Ozin, J. Am. Chem. Soc. 122 (2000) 8932

[3] Z. Feng, W.S. Postula, C. Erkey, C.V. Philip, A. Akgerman, R.G. Anthony, J. Catal. 148 (1994) 84.

[4] D.E. Collins, K.A. Rogers, K.J. Bowman, J. Eur. Ceram. Soc. 15 (1995) 1119.

[5] C. Li, L. Yamai, Y. Murase, E. Kato, J. Am. Ceram. Soc. 72 (1989) 1479.

[6] D.G. Lamas, G.E. Lascalea, N.E. Walsöe de Reca, J. Eur. Ceram. Soc. 18 (1998) 1217

[7] M.P. Pileni, J. Phys. Chem. 97 (1993) 6961.

[8] M.H. Lee, C.Y. Tai, Y.C. Wu, Chem. Eng. Sci. 56 (2001) 2389.

[9] J. Wang, L.S. Ee, S.C. Ng, C.H. Chew, L.M. Gan, Mater. Lett. 30 (1997) 119.

[10] C.Y. Tai, B.-Y. Hsiao, H.-Y. Chiu, Colloids Surf., A Physicochem. Eng. Asp. 237 (2004) 105.

[11] C.Y. Tai, B.-Y. Hsiao, Chem. Eng. Commun. 192 (2005) 1525.

[12] N.L. Wu, S.Y. Wang, I.A. Rusakova, Science 285 (1999) 1375.

[13] N. Lufimpadio, J.B. Nagy, E.G. Derouane, in: K.L. Mittal, B. Lindman (Eds.), Surfactants in Solution, vol. 3, Pleum Press, New York, 1984, p. 1483

[14] Z.G. Wu, Y.X. Zhao, D.S. Liu, Microporous Mesoporous Mater. 68 (2004) 127.

[15] F. del Monte, W. Larsen, J.D. Mackenzie, J. Am. Ceram. Soc. 83 (2000) 1506

[16] M.J. Torralvo, M.A. Alario, J. Soria, J. Catal. 86 (1984) 473. 